
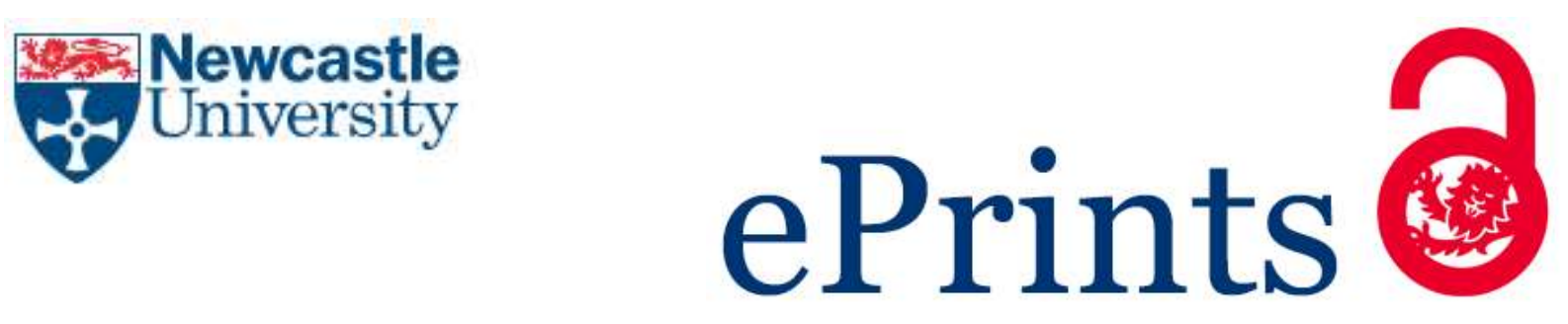

Dade-Robertson M. Building Science: Synthetic Biology and emerging technologies in architectural research. Architectural Research Quarterly 2016, 20(01), 5-8.

\title{
Copyright:
}

This is the peer reviewed version of the following article: Dade-Robertson M. Building Science: Synthetic Biology and emerging technologies in architectural research. Architectural Research Quarterly 2016, 20(01), 5-8., which has been published in final form at http://dx.doi.org/10.1017/S1359135516000142 This article may be used for non-commercial purposes in accordance with Wiley Terms and Conditions for Self-Archiving.

Date deposited:

$01 / 08 / 2016$

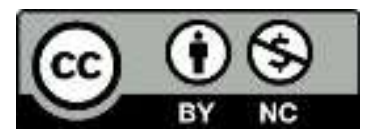

This work is licensed under a Creative Commons Attribution-NonCommercial 3.0 Unported License 


\title{
Building Science: Synthetic Biology and emerging technologies in architectural research
}

\author{
Martyn Dade-Robertson
}

Behind the main Architecture building at Newcastle University, UK, is a squat 1960s extension with a plaque that reads 'Building Science'. The building no longer contains a Building Science department but instead graduate design studios. There are reminders of the building's heritage in the 'Test Chamber' (an unfortunate title for what has become a crit space), a double height room with chains hanging ominously from the ceiling. However, even when I was a student at Newcastle in the late 1990s, Building Science in the school had begun to change into something predominantly conducted on computers or elsewhere in the University by civil engineers. This decline is in contrast to Building Science and Architectural Science research which has thrived elsewhere in other institution but has given us an opportunity to look again at what we mean by Building Science.

I was recently involved in a bid for research funding and, as part of the initial pitch, I presented the panel with a small plastic pot containing a liquid culture of approximately eight billion $E$. coli bacteria cells. I proposed that the content was more than a smelly broth. It was, rather, a combination of eight billion architects, civil engineers and construction workers. I went on to describe a Synthetic Biological system in which genetically engineered bacteria cells could be suspended in a saturated aggregate and, by sensing increased loads, could respond by synthesising materials to strengthen the aggregate where it was needed. During the follow-up Q\&A I was asked 'what level of technological readiness would my demonstrator would be at by the end of the project'. I had no idea what constituted 'a level of technological readiness'. In a similar situation I presented a version of this project at a job interview. I was asked, by the rather bemused interviewer, whether this was more of a material science problem with little, as yet, to do with architecture. I again didn't have a suitable response. After my funding pitch I was concerned 
enough to Google 'Level of Technological Readiness' but what I found only confused me further.

\section{Technological readiness}

The Technological Readiness Level (TRL) was developed in the US and is used in various forms by government agencies such as NASA and the EU. The model illustrated in [1] is a version taken from the European Commission's Horizon 2020 work programme. There are various versions for different domains but its structure is based on a series of nine stages starting with basic research into fundamental principles (TRL 1) to 'system- proven' technologies in 'operational environments' (TRL 9). The TRL treats the maturity of technology in terms of its relationship to the environment of deployment and as a simple linear progression from concept to industrial application. Early research is conducted in theoretical - often computational models and then demonstrated in controlled lab environments before being deployed and tested in the real world and becoming 'industrially relevant' only at TRL 5. Seen in this context, any form of research which could be considered Building Science in architecture would naturally tend towards the bottom of the chart. We, as architects, are concerned with the real world, with what we can build. As architects we accept the wealth of materials and technologies provided by science and engineering and focus on their innovative repurposing and configuration in the messy and complex built environment. This might sum up much Building Science research, wedged between a conservative and risk averse building industry and the need for buildings which are more radically efficient using a series of technologies, including new materials and digital sensing \& fabrication systems which are often developed, in the first instance, for other industries and applications.

My project, however, certainly didn't come anywhere near the bottom of the TRL. My project came from a different place. To some extent, the proposition had more in common with speculative or experimental architecture than it did with scientific or engineering design. What I didn't mention in my funding interview was that the idea for a bacteria-based material capable of responding to mechanical changes had originally been developed as a paper which speculated on the colonisation of Mars. ${ }^{1}$ The paper was more provocation than science and was written to appeal to science fiction writers as much as to NASA. In writing the paper and visualising our design proposals, we were participating in a tradition of architectural speculation. Look at the degree shows of many of the more progressive schools of architecture and you will find plenty of speculative projects involving a range of biotechnologies, nanotechnologies, advances in neuroscience etc. However, by asking for funding to develop the research I was looking to do something beyond speculation. My project proposal represented an attempt to make a speculation into a grounded reality, which 
is perhaps the essence of design. However, as a research project the proposal couldn't hope to achieve the speculative vision and, indeed, may produce evidence of how far out our speculation was.

We are now entering an era where biotechnology is, we are told, about to have a transformative effect on society comparable with the industrial and digital revolutions ${ }^{2}$. The speculative discourse in architecture has been quick to catch on to this, and one of the editors and one of the contributors to this issue of arq have already edited issues of $A D$ covering a range of biotechnologies. ${ }^{3}$ However, arq is a different kind of journal from $A D$. Set up to bridge academic research and practice, arq has traditionally taken a more pragmatic approach to its engagement with emerging technologies preferring to publish on real outcomes rather than illustrating broad speculation. By proposing this special issue on Biotechnology and the Built Environment we are challenging arq's thematic focus.

This issue becomes even more challenging if we take a subfield of biotechnology - my chosen specialism of Synthetic Biology. Synthetic Biology is an emerging field which has been embraced by government as one of the Eight Great Technologies ${ }^{4}$ set to transform society and therefore subject to substantial UK (and international) research investment. It is also a fledgling discipline and one which remains contested. Synthetic Biology is increasingly associated with the application of engineering design methods to the design of biological systems and particularly emphasises genetic manipulation. ${ }^{5}$ An important research question is how to design and engineer systems at the level of individual molecules to influence systems at the building or even city scales. Before last year, when I embarked on a post-graduate degree in Synthetic Biology, I would have described the state of the art in Synthetic Biology with reference to computing in the 1960s and '70s which was then poised to have a transformative effect on society. I rationalised my decision to pursue a degree in the field as the equivalent to an architect undertaking studies in Computer Science in anticipation of the CAD revolution in the coming decades. I was, in effect, future proofing myself. If I were to draw the same parallel now, however, I would describe Synthetic Biology as being at its Babbage stage - with foundational concepts forming but with few practical demonstrators yet and fundamental questions remaining. On that basis, I might have taken my Synthetic Biology degree two hundred years too early. Plotted on the TRL chart, most Synthetic Biology demonstrators can be considered at, or close to, the top.

\section{Synthetic biology: an alternative Building Science}

Perhaps then it is too early for architects to be interested in Synthetic Biology. Or perhaps not. I want to make the claim here for an alternative (and additional) sort of Building Science. A Building Science which is not necessarily concerned with the pragmatic construction of the built 
environment but which helps develop experiments that support the design of real systems and goes beyond futurism and speculation. I'm proposing a type of Building Science which not only engages with technologies at the bottom of the TRL but which also supports the development of a discourse of scientific research which is overt in its 'speculative' focus. Synthetic Biology, it seems to me, is a good test for this new type of Building Science. This emerging discipline aims to secure understanding, not only through analysis but also through synthesis, and reviews of Synthetic Biology often include the Richard Feynman quote 'What I cannot create, I do not understand'. ${ }^{6}$

This closer alignment between architectural and early stage scientific research would not have been a strange concept to, for example, Wren or Buckminster Fuller. The invention by Fuller of the tensegrity structure paralleled its discovery as the major structural system used by many types of Cell to retain their morphology.7 It seems, at least anecdotally, that the spatial and visual capacity of architects - both in terms of interpretation and synthesis - has something to offer biological understanding.

From another perspective it feels as if architecture could also benefit from collaboration with early stage scientific research. In the twenty-first century much of the most prominent architectural research in computing is centred on robotics. Architecture schools have been buying robotic arms to shape materials and even to lay bricks. ${ }^{8}$ What is curious about these research enterprises is that they make use of technology which was available in Japanese car plants in the 1960s - indeed sometimes exactly the same hardware. These robots, which are typically fixed robot arms, are hacked to add or subtract material as long as the space of operation is within their reach. What if, as robots for the automotive industry had been developed in the 1960s, Building Scientists had also begun to work in robotics. Perhaps they would have challenged robotics to produce different form factors and mechanical systems relevant to the messy and uncertain world of the building site? I'm almost certain that they wouldn't have come up with a robot arm as the preferred option.

\section{Scientific collaborations}

In attempting to apply this aspiration for more foundational scientific collaboration between Architecture and Synthetic Biology my concern has been to look for ways in which my research group could devise both provocative and challenging applications and turn those provocations into scientific questions. I can't pretend to have a rigorous model for the operation of this 'method'. However, I can offer a personal anecdote. After presenting the aforementioned pot of bacteria to the funding panel I was (to my surprise) able to obtain funding for my pressure sensing and aggregating bacteria. My pitch had focused on the idea of a self-forming foundation. A building would be constructed on a weak soil laced with my engineered bacteria which, in 
response to pore pressure increases as the soil is loaded, would rapidly synthesise material to help glue the soil together and resist the load. This idea is, in part, biomimetic and refers to the way in which biological systems, from the synthesis of bone to the stem cells in plants, respond to mechanical changes in their environment. These processes have been of interest to architectural design, most notably through the work of Neri Oxman who uses a material based computation approach to sculpt materials into patterns which are structurally efficient and based on the properties of the materials used. ${ }^{9}$ However, biological systems of materials synthesis and response don't separate computation, modelling and material synthesis but combine them in living cells.

We initially broke the project down into component parts - the first of which was to identify a mechanism for pressure sensitivity in bacteria. It is a mark of our naivety that we assumed that such a mechanism existed and it became evident that this went against received wisdom in the scientific community. ${ }^{10}$ Our assumption, which we turned into a hypothesis, was that bacteria do (indeed must) respond genetically to even low levels of pressure given the inevitable forces which would be placed on the bacterial cell wall. To test this we pressurised the bacteria and measured the amount of RNA expressed in both normal and pressurised conditions (we chose 10atm). We discovered a genetic response in almost one hundred and fifty genes. This meant that our bacteria were sensing moderate changes in environmental pressure at the level of their genetics - responding by making or arresting production of different types of protein. These are early results and, even if verified, do not constitute Nobel Prize winning discoveries. They do, however, constitute publishable research. Not, of course, architectural research. At least, not yet.

In parallel we have used this data to begin to speculate about a design process involving our hypothetical pressure sensing bacteria. Starting with the idea of making biologically synthesised foundations using our pressure sensitive bacteria it became clear that, because gene expression is rarely linear, we would not expect to find a gene which is increasingly expressed in a linear fashion as pressures rise. Different gene regulators have different sensitivities. Furthermore, as part of a system in which a gene for pressure sensitivity may communicate to another genes (or even another cell) the dynamics of this system become increasingly complex. When we conducted this research we needed to use hypothetical (although still grounded) values for gene expression to produce visualisations which give an indication for the amount of consolidation throughout the soil matrix) [2]. The results are, from one perspective, problematic because our biological foundations would be liable to fail - forming some way underneath the buildings base or developing unconsolidated voids at their core. For me, however, the visualisations provided a much more promising (if tentative) direction. Rather than 
considering synthetic consolidation as part of a stabilising system for foundations, it may be better to consider it as part of a material sculpting system. In the visualisations I observed vaults and caverns many metres across being sculpted through the (potentially very slight) manipulation of the gene code of a micro-organism. The simulation software we produced offered an alternative CAD system - in which the synthesis of form was the result of altering patterns of gene expression.

\section{Speculations and intuitions}

When I have presented this project to an audience of microbiologists, words such as 'speculation', 'intuition' and the use of 'hypotheticals' tend not to go down well. To an audience of civil engineers, the concepts have often (although not always) been considered meaningless without hard data and without a clear problem to solve. That our visualisations seem to show that our system would fail in the context for which it was designed is seen as an indication that our experiment has also been a failure. This work clearly does not fit onto a TRL diagram as it jumps from basic scientific research to (hypothetical) environmental implementation. However, in contrast, when presenting this work to architectural or general design audiences, I have the permission to mix scientific investigation with design projection and grounded speculation. The work gets judged, at least in part, by the quality of the ideas and the creativity of exploration. We simply don't have the same constraints in architecture as in other fields of research and there is, I believe, and opportunity to exploit this freedom. We may need a new TRL diagram which shows that at least some of the 'implementation' takes place only in the form of design projection.

Retuning to the question of what 'Building Science' means, work on Synthetic Biology provides an interesting starting point and, to some extent, a counterpoint to traditional Building Science research. Whereas there are now established fields of research in architecture related to areas of, for example, digital technologies, fabrication and robotics there is no such established research program in architecture related to the biological sciences (although these are emerging). Because the scientific work in areas such as Synthetic Biology is still relatively new, our engagement with them has yet to be defined. We can wait until the first generation of technologies gets past stage 1 or 2 or 3 or 8 of the TRL when we can design and build real buildings and systems or we can try to help define and engage in fundamental research now, well before these technologies are 'ready'. The latter approach requires us to be speculative and to accept that these speculations may be sacrificial - a way of defining concepts rather than providing a robust solution for a particular application domain. However, it also forces us to ground those speculations in real concepts and experimentations and collaborations with people and disciplines some distance away from traditional architectural 
research. In this way the 'Building' in 'Building Science' will, for architecture, become a verb as well as a noun.

Martyn Dade-Robertson is a Senior Lecturer in Architecture at Newcastle University where he specialises in design computation with an interest in emerging technologies, particularly Synthetic Biology. He holds degrees in architectural design, architectural computation and synthetic biology and is the author of over 20 peer reviewed publications including the book The Architecture of Information published by Routledge in 2011.

\section{Acknowledgements}

I would like to thank Newcastle University's School of Architecture Planning and Landscape, Centre for Cell and Bacterial Biology, School of Computer Science and School of Chemistry for their support in this highly interdisciplinary endeavor. In addition I would like to thank Anil Wipat, Wendy Smith, Meng Zhang, Steven Robertson, Christine Dade-Robertson, the residents of Level 3 at CBCB and the students of the BioComp MSc. and Adam Sharr. The Computational Colloids project described here is kindly supported by the EPSRC - Grant number: EP/N005791/1.

\section{Notes}

1. Dade-Robertson, M, M Zhang, and C Ramirez-Figueroa, 'Radical Vernacular: Bacterial Architecture on Mars', Journal of the British Interplanetary Society, 2015

2. Voigt, Christopher, Synthetic Biology Scope, Applications and Implications (London: Royal Academy of Engineering, 20 January 2012)

3. Cruz, Marcos, and Steve Pike, eds., Neoplasmatic Design (Chichester: Wiley and Sons, 2008); Spiller, N, and Rachel Armstrong, Protocell Architecture (Chichester: Wiley and Sons, 2011)

4. Willetts, David, Eight Great Technologies (London: Policy Exchange, 2013)

5. Heinemann, Matthias, and Sven Panke, 'Synthetic Biology-Putting Engineering into Biology.', Bioinformatics (Oxford, England), 22 (2006), 2790-9

6. O'Malley, Maureen a., 'Making Knowledge in Synthetic Biology: Design Meets Kludge', Biological Theory, 4 (2009), 378-389

7. Davies, Jamie, A., Mechanisms of Morphogenesis (London: Elsevier, 2005)

8. Kolarevic, B, and K Klinger, eds., Manufacturing Material Effects: Rethinking Design and Making in Architecture (London: Routledge)

9. Oxman, Neri, Steven Keating, and Elizabeth Tsai, 'Functionally Graded Rapid Prototyping', in Innovative Developments in Virtual and Physical Prototyping, 2011, pp. 483-490 
10. Follonier, Stéphanie, Sven Panke, and Manfred Zinn, 'Pressure to Kill or Pressure to Boost: A Review on the Various Effects and Applications of Hydrostatic Pressure in Bacterial Biotechnology', Applied Microbiology and Biotechnology, 93 (2012), 1805-181

\section{CAPTIONS:}

\section{Figure 1}

Diagram to illustrate the Technology Readiness Level based on the table used by the EU Horizon 2020 program.

\section{Figure 2}

Diagrams to show the patterns of consolidation in a soil volume of $10 \mathrm{~m} \mathrm{x}$ $10 \mathrm{~m}$. The diagram shows the results of different expression profiles for bacterial with two different pressure sensitivities in the soil. The soil volume is shown both whole and in section.

\section{WEB ABSTRACT (300 words):}

The paper examines the concept of Building Science through the role of emerging scientific research and technologies. The paper takes as its starting point the Technology Readiness Strategy which is a way of judging the state of a technology in terms of its readiness for environmental deployment and relevance to industry. The paper argues that this model is limited and uses the example of Synthetic Biology to argue for a type of building science which is both speculative and grounded and which may not lead to immediate or short-term applications but is driven by hypothetical contexts and imagined futures. The paper argues that both scientific knowledge and architectural research may be enhanced through deeper collaboration and gives the example of a project to develop a genetically engineered mechanical sensing bacteria capable of making materials which respond to physical forces in their environment. The paper suggests that, while initiated with an application domain in mind, the knowledge gained from the project has pointed to alternative avenues for creative design explorations. The paper concludes that an alternative form of Building Science may be possible in which the term 'building' is both a verb and a noun. 